\title{
Effects of Orthokeratology on Axial Length Elongation in Anisometropes
}

\author{
Jing $X^{a, b}$ Bei Gao ${ }^{a}$ Qingmei Tian ${ }^{b, c}$ Qiuxin Wu ${ }^{b, c}$ Xiuyan Zhang ${ }^{b, c}$ \\ Xiao Lin ${ }^{a}$ Ruixue Zhang ${ }^{a}$ Jike Song ${ }^{a, c}$ Hongsheng $\mathrm{Bi}^{\mathrm{b}, \mathrm{c}}$
}

aShandong University of Traditional Chinese Medicine (TCM), Jinan, PR China; ${ }^{b}$ Affiliated Eye Hospital of Shandong University of TCM, Jinan, PR China; 'Shandong Provincial Key Laboratory of Integrated Traditional Chinese and Western Medicine for Prevention and Therapy of Ocular Diseases, Key Laboratory of Integrated Traditional Chinese and Western Medicine for Prevention and Therapy of Ocular Diseases in Universities of Shandong, Eye Institute of Shandong University of TCM, Jinan, PR China

\section{Keywords}

Orthokeratology · Myopia · Anisometropia · Retrospective study

\begin{abstract}
Introduction: This study aimed to investigate the therapeutic effects of overnight orthokeratology (OK) lenses on anisometropes. Methods: We enrolled 178 anisometropes from August 2015 to August 2017. We then divided these patients into 2 parts depending on them wearing either monocular or binocular OK lenses. In part one, 47 monocular myopic subjects ( 25 males and 22 females) were treated with OK lenses in the myopic eyes only. We also labeled the myopic eyes as the OK group and the contralateral nonmyopic eyes as the control group. The initial average wearing age of the subjects was $12.35 \pm 2.37$ years (8-16 years). The mean follow-up duration was $15.43 \pm 4.88$ months (7-25 months). The average spherical equivalent refraction (SER) was $-2.31 \pm 1.16$ diopter (D) in the OK group and $0.15 \pm 0.49 \mathrm{D}$ in the control group $(p<0.001)$. In part 2, 131 binocular myopic anisometropes (56 males and 75 females) were involved in the study. The eyes with more severe myopia were assigned to the $\mathrm{G}$ group and the contralateral eyes to the $L$ group. The initial average wear-
\end{abstract}

(C) 2021 The Author(s)

Published by S. Karger AG, Basel

This is an Open Access article licensed under the Creative Commons Attribution-NonCommercial-4.0 International License (CC BY-NC) (http://www.karger.com/Services/OpenAccessLicense), applicable to the online version of the article only. Usage and distribution for commercial purposes requires written permission. ing age of the subjects was $12.92 \pm 2.60$ years ( $8-16$ years). The mean follow-up duration was $17.83 \pm 5.02$ months (7-26 months). The average SER was $-4.79 \pm 1.90 \mathrm{D}$ in the $\mathrm{G}$ group and $-3.14 \pm 1.88 \mathrm{D}$ in the $\mathrm{L}$ group $(p<0.001)$. We calculated the axial length $(A L)$ difference and $A L$ elongation as our primary outcome measures. Results: In part one, the AL elongation in the OK group $(0.21 \pm 0.09 \mathrm{~mm})$ was significantly lower than that in the control group $(0.70 \pm 0.17 \mathrm{~mm})$ at 24 months $(p<0.001)$. Meanwhile, the AL difference exhibited a decrease of $0.50 \pm 0.29 \mathrm{~mm}$ from a baseline of $1.08 \pm 0.35$ to $0.58 \pm 0.25$ mm at 24 months $(F=24.539, p<0.001)$. In part 2 , the $A L$ had increased by $0.17 \pm 0.13 \mathrm{~mm}$ in the $\mathrm{G}$ group and $0.24 \pm 0.18$ $\mathrm{mm}$ in the $\mathrm{L}$ group after 24-month follow-up, respectively $(p<0.001)$. While the AL difference decreased from $0.55 \pm 0.11$ $\mathrm{mm}$ at the baseline, to $0.48 \pm 0.08 \mathrm{~mm}$ at 24 months, eliciting a decrement in $\mathrm{AL}$ difference of $0.07 \pm 0.09 \mathrm{~mm}(F=3.884$, $p=0.030)$. Conclusions: OK lenses can slow down AL growth in anisometropes and has a greater effect on reducing $\mathrm{AL}$ elongation in the more severely affected myopic eyes of anisometropic patients.

(C) 2021 The Author(s) Published by S. Karger AG, Basel

Jing Xu and Bei Gao contributed equally.
Correspondence to:

Jike Song, edusjk@163.com

Hongsheng Bi, hongshengbil@163.com 


\section{Introduction}

Anisometropia is a condition in which the refractive error differs between the 2 eyes. A spherical equivalent refraction (SER) discrepancy of 1.00 diopter (D) or above between both eyes is usually implemented as the definition of anisometropia [1]. Anisometropia can lead to diplopia, aniseikonia, decreased stereopsis, visual fatigue, and even amblyopia in the more myopic eye. These symptoms usually become obvious when the degree of anisometropia is $2.50 \mathrm{D}$ or more [2-4]. High degrees of anisometropia could lead to a significant difference in the retinal image size and cause asthenopia, amblyopia, and stereopsis impairment $[5,6]$. Anisometropia is a rather rare condition in the general population. Prevalence estimates of anisometropia vary in different studies and may be dependent on age, inclusion criteria, and the definition of anisometropia. Ethnic or racial differences in anisometropia prevalence have not been studied directly [7]. Previous cross-sectional studies showed that the prevalence of anisometropia is age-dependent, with a relatively low prevalence (1.6-4.3\%) among young children and a higher prevalence in adults [7-10]. A few longitudinal studies reported that the chance of developing anisometropia increases after children start school. A study in Japan found a slight increase in the prevalence of spherical anisometropia (spherical difference $\geq 1.00 \mathrm{D}$ ), rising from $1.43 \%$ at 6 years old to $3.14 \%$ at 11 years old [11]. The tendency of higher anisometropia prevalence with increasing age continues after the age of 60 years, climbing from about $10 \%$ for 60 - to 69 -year olds to $>30 \%$ for individuals aged $80+$ years [12-14]. In older people, anisometropia might be due to differential changes in lens power and development of cataracts.

Even though there is substantial evidence of the axial nature of anisometropia, little is known about what initially triggers mismatched eye growth [15-17]. Most cases of anisometropia appear to be axial in nature, as demonstrated in studies of both animal and human eyes reporting a positive correlation between the degree of anisometropia and the interocular axial length $(\mathrm{AL})$ difference [15-18]. Abnormal visual input in 1 eye may cause more AL elongation in that eye, as demonstrated in animal studies of various species $[15,16,19]$. Defects in the structure of the optical components due to prematurity [20,21] or cataracts [22] could also result in significant refraction differences.

Interventions [5, 23-25] that have been attempted for this condition include glasses, multifocal lenses, multifocal contact lenses, atropine, and surgery. However, de- spite providing an amelioration of the patients' vision, these treatments do not provide adequate myopia control effects, and the magnitude of myopic anisometropia might even increase over time. On the other hand, lower degrees of anisometropia are usually well tolerated with glasses, and children with higher degrees of anisometropia ( $\geq 4.00 \mathrm{D})$ are more likely to develop amblyopia during their preschool years making symptomatic management considerably more difficult to achieve [26]. Refractive surgery has been suggested as a possible treatment strategy for such patients to minimize aniseikonia and improve visual acuity [27-29]. Nonetheless, because the onset and progression of such large differences in refraction between the 2 eyes are not clearly known, the choice of the age at surgery and accurate interpretation of the refractive stability after refractive surgery is always biased by the possible age-related refractive changes in that particular age-group.

Orthokeratology (OK) lenses are rigid contact lenses with a reverse geometry on the posterior surface [30,31]. Overnight wear of the lens flattens the central cornea zone and increases the relative corneal refractive power in the periphery. This inverted pattern of corneal peripheral refraction induces a relative peripheral myopic defocus on the retina, which is considered the working mechanism of the OK lenses [32-34]. The potential of OK lenses for myopic correction and control has been confirmed by several studies [25, 35-37]. Interestingly, OK lenses have demonstrated a beneficial effect against the progression of anisomyopia [38]. Wearing OK lenses for a long period of time would present a more significant AL difference reduction in children with severe anisometropia $[39,40]$.

This study is a retrospective study focusing on anisometropic children. The main purpose of this study was to analyze the effectiveness of the OK lens on the anisometropia in children.

\section{Methods}

Subjects

In this retrospective study, all the subjects $(n=178)$ were obtained from the Optical Center of the Affiliated Eye Hospital of Shandong University of Traditional Chinese Medicine (Shandong, China) from August 2015 to August 2017. The pertinent data depicted from examination results were retrieved and rearranged from the medical records of children who used OK lenses for vision correction.

Depending on wearing monocular or binocular OK lenses, the patients were divided into 2 parts (part one: only myopic eye with OK lens and part 2: binoculus with OK lens). In part one, we re- 
viewed 47 monocular myopic children ( 25 males and 22 females); the myopic eyes treated with $\mathrm{OK}$ lenses were divided into the OK group and the contralateral eyes were divided into the control group. In part 2, 131 myopic anisometropes (56 males and $75 \mathrm{fe}-$ males) were involved in the study, and the eyes with greater degrees of initial myopia were assigned to the G group and those with less initial myopia were assigned to the L group.

The participants who were wearing the OK lenses of Mengdaiwei (Mengdaiwei, Sino-American joint) were enrolled in the study. The OK lenses were spherical 4-zone lenses made of gas-permeable lens material (Boston XO). Three practitioners of the Optical Center adopt the same guideline to perform the OK. The study was conducted in accordance with the principles of the Declaration of Helsinki, and the study protocol was approved by the Ethics Committee of the Affiliated Eye Hospital of Shandong University of Traditional Chinese Medicine. Because of the retrospective nature of the study, patient consent for inclusion was waived.

\section{Inclusion Criteria}

1. Aged between 8 and 16 years; initial anisometropia in cycloplegic SER above $1.00 \mathrm{D}$

2. The SER in the myopic eye being between $-6.00 \mathrm{D}$ and -1.00 $\mathrm{D}$, and the nonmyopic eye being between -0.50 and $+1.50 \mathrm{D}$; with-the-rule astigmatism $>-1.50 \mathrm{D}$

3. The myopic eyes had to have uncorrected visual acuity lower than $20 / 25$ and monocular corrected visual acuity exceeding 20/20. Meanwhile, the nonmyopic eyes had to have uncorrected visual acuity of $20 / 25$ or better so make sure that no correction was needed

4. $10-21 \mathrm{~mm} \mathrm{Hg}$ of intraocular pressure (IOP)

5. Had at least 2 AL recordings, including values at baseline and final follow-up visit

\section{Exclusion Criteria}

1. Patients with other eye diseases and prior surgery, as well as ocular and systemic conditions that might affect vision or a history of using atropine, RGP, and OK lenses to control the progression of myopia

2. Have significant deviations during lenses wear

\section{Measurements}

Data collected from the patient's files consisted of the participant's demographic and visual parameters. Cycloplegic autorefraction was performed after the instillation of 4 drops of compound tropicamide eye drops $(0.5 \%$ tropicamide and $0.5 \%$ neosynephrine) (Santen, Japan) separated 10 min apart in each of the patients' eyes. Ten minutes after the instillation of the fourth drop, autorefraction measurements were taken by auto-refractor NIDEK (ARK-700A; NIDEK; JA-PAN); the IOP from the noncontact tonometer (NIDEK NT-510; NIDEK; JA-PAN); the corneal diameter (CD), pupil diameter (PD), keratometry, anterior chamber depth (ACD), and AL from the IOL-Master (V5.0; Carl Zeiss Meditec AG; Jena; Germany). For each measurement, 3 successive measurements were subsequently calculated with their mean data as representative values.

We calculated the AL difference (the AL of the more severe myopic eye minus that of the contralateral eye) and AL elongation as our primary outcome measures. We measured the AL and AL elongation of eyes at $0,6,12,18$, and 24 months $(x \pm 2$ months at each time point were included).

Effects of Orthokeratology on

Anisometropes

\section{Statistical Analysis}

SPSS software (version 22.0; IBM, USA) was used to perform statistical analysis. $p=0.05$ was used to denote statistical significance. The data distribution was assessed using the Shapiro-Wilk test prior to performing parametric tests. The data exhibited a normal distribution (Shapiro-Wilk test, $p>0.05$ ) and were presented as the mean \pm standard deviation. Subsequently, independent $t$ tests were used to compare the baseline SER, keratometry, ACD, $\mathrm{PD}, \mathrm{CD}, \mathrm{IOP}, \mathrm{AL}$ of the 2 groups, and AL elongation of 2 eyes at different follow-up times. Multiple imputation was used for handling missing values in longitudinal data and repeated-measures ANOVA was performed to assess the changes of AL difference over time. Generalized estimating equation (GEE) was used to evaluate the correlation between parameters and AL elongation during the follow-up period, the associations between the change of AL difference and other parameters. (follow-up time: baseline, $6,12,18$, and 24 months).

\section{Results}

\section{Part One}

Patient Characteristics with Monocular OK Lenses

We reviewed the data of 47 patients who used monocular $\mathrm{OK}$ lenses. The average initial wearing age was $12.35 \pm 2.37$ years (from 8 to 16 years), and the mean follow-up duration was $15.43 \pm 4.88$ months (from 7 to 25 months). Additionally, the average initial AL was significantly longer in the OK group $(24.54 \pm 0.94 \mathrm{~mm})$ than that in the control group $(23.46 \pm 0.74 \mathrm{~mm})(p<0.001)$. The average SER was $-2.31 \pm 1.16 \mathrm{D}$ in the $\mathrm{OK}$ group and $0.15 \pm 0.49 \mathrm{D}$ in the control group $(p<0.001)$.

There was no statistical difference in the initial keratometry, ACD, PD, CD, and IOP. The baseline biometric data for the $\mathrm{OK}$ and control groups obtained immediately before the children began wearing the lenses are illustrated in Table 1.

\section{The AL Elongation in the OK Group Was}

Significantly Slower than That in the Control Group

In the OK group, the initial $\mathrm{AL}$ was $24.54 \pm 0.94 \mathrm{~mm}$ and then $24.74 \pm 0.87 \mathrm{~mm}(p<0.001)$ after 2 years of follow-up. In the control group, the AL increased from 23.46 \pm 0.74 to $24.16 \pm 0.69 \mathrm{~mm}(p<0.001)$. During the 2 -year follow-up period, the control group exhibited more change in $\mathrm{AL}$ than that of the OK group. In the OK group, the $\mathrm{AL}$ elongation was $0.06 \pm 0.11 \mathrm{~mm}$, and the control group elicited higher AL elongation values of up to $0.43 \pm 0.15 \mathrm{~mm}$ at 12 months $(p<0.001)$. At 24 months of follow-up, the AL elongations were $0.21 \pm 0.09$ and $0.70 \pm 0.17 \mathrm{~mm}$ in the OK group and control group $(p<0.001)$, respectively. Compared to the $\mathrm{OK}$ group, there was a significant $\mathrm{AL}$ elongation in the control group (Table 2; Fig. 1).

Ophthalmic Res 2021;64:991-1001 
Table 1. Summary of the demographic data of the OK group and the control group

\begin{tabular}{lccr}
\hline Variables & OK group & Control group & $p$ value \\
\hline Gender, $n(\%)$ & & & \\
$\quad$ Male & $25(53.2)$ & & \\
$\quad$ Female & $22(46.8)$ & & \\
Wearing age & $12.35 \pm 2.37(8-16$ years $)$ & & \\
Follow-up time & $15.43 \pm 4.88(7-25$ months $)$ & & \\
Side & & & \\
$\quad$ Right & 36 & 11 & $<0.001^{*}$ \\
$\quad$ Left & 11 & 36 & $0.908^{*}$ \\
SERE, D & $-2.31 \pm 1.16$ & $0.15 \pm 0.49$ & $0.635^{*}$ \\
K1, D & $42.57 \pm 1.44$ & $42.54 \pm 1.52$ & $0.357^{*}$ \\
K2, D & $43.80 \pm 1.62$ & $3.69 \pm 1.47$ & $0.614^{*}$ \\
ACD, mm & $3.66 \pm 0.19$ & $5.99 \pm 0.86$ & $0.966^{*}$ \\
PD, mm & $5.99 \pm 0.90$ & $12.10 \pm 0.32$ & $0.905^{*}$ \\
CD, mm & $12.11 \pm 0.33$ & $16.42 \pm 2.73$ & $<0.001^{*}$ \\
IOP, mm Hg & $16.66 \pm 2.44$ & $23.46 \pm 0.74$ & \\
SERE, D & $24.54 \pm 0.94$ & & \\
\hline
\end{tabular}

SER, spherical equivalent refractive; $\mathrm{K} 1$, the refraction measured on the flattest curve of the cornea; K2, the refraction measured on the deepest curve of the cornea; ACD, anterior chamber depth; PD, pupil diameter; $\mathrm{CD}$, corneal diameter; IOP, intraocular pressure; $\mathrm{AL}$, axial length; $\mathrm{mm} \mathrm{Hg}$, millimeter of mercury; $\mathrm{OK}$, orthokeratology; $\mathrm{D}$, diopter. Data are presented as $n(\%)$ or mean standard \pm deviation. $p$ value $<0.05$ was considered statistically significant after the test. ${ }^{*} p \leq 0.05$ (obtained from independent $t$ tests).
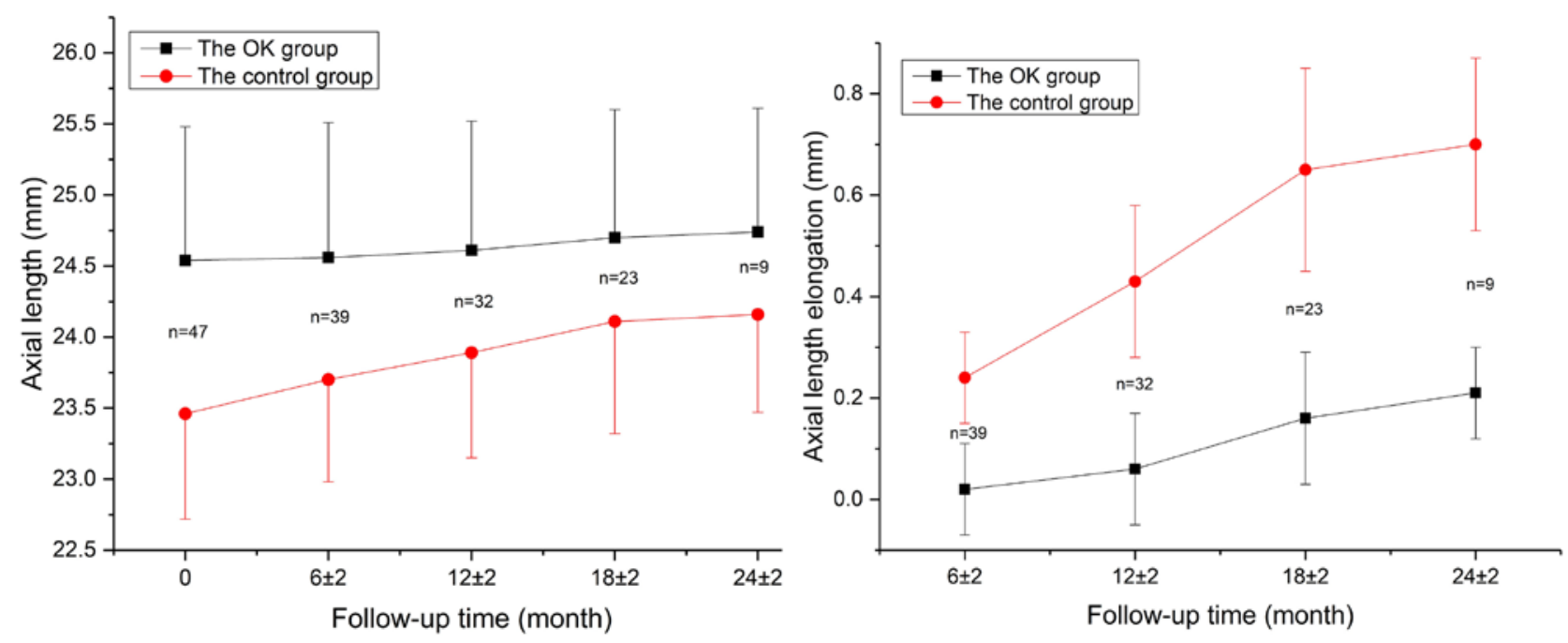

Fig. 1. Changes in AL and AL elongation of the OK group and control group. Error bars represent standard errors. AL, axial length; OK, orthokeratology.

In the GEE model, the variable factors of gender, keratometry, ACD, IOP, PD, CD, SER, and age exhibited no effect on the AL elongation ( $p>0.05)$. While, wearing OK lens and follow-up time exhibited effect on the AL elongation $(p<0.05$, Table 3$)$.
OK Lenses Significantly Decreased the AL Difference During the study period, the AL difference considerably declined. After 12 months of treatment, the AL difference decreased by $0.36 \pm 0.25 \mathrm{~mm}$, from $1.08 \pm 0.35$ $\mathrm{mm}$ at baseline to $0.71 \pm 0.30 \mathrm{~mm}$ at the 12 -month visit. 
Table 2. The AL, AL elongation, and AL difference in the OK group and control group

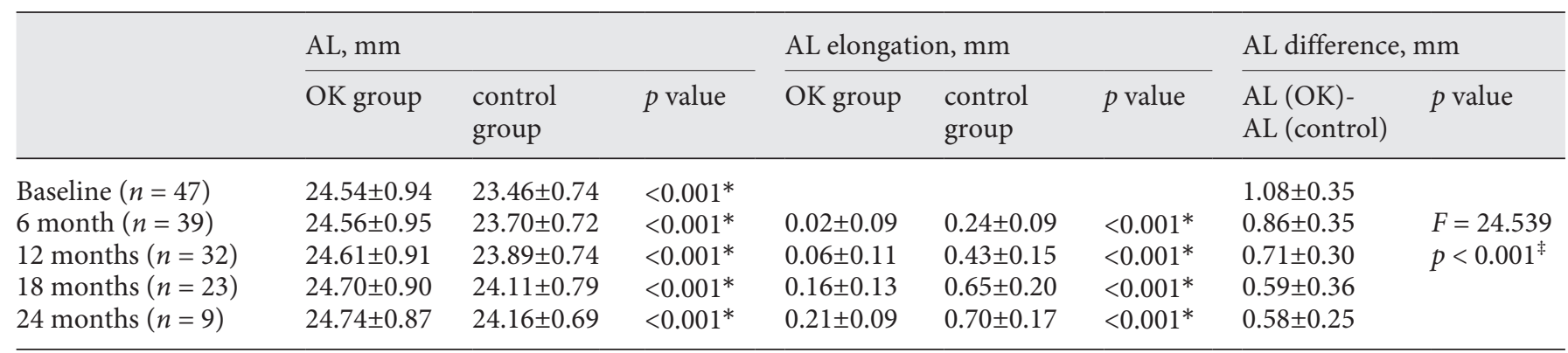

OK, orthokeratology; ANOVA, analysis of variance; AL, axial length. $p$ value $<0.05$ was considered statistically significant after the test. ${ }^{*} p$ obtained from an independent $t$ test. ${ }^{*} p$ obtained from repeated-measures ANOVA.

Table 3. The associations between variable factors and AL elongation during the period of follow-up

\begin{tabular}{|c|c|c|c|c|c|c|c|c|}
\hline Predictors & $\beta$ & $95 \% \mathrm{CI}$ & $\exp (\beta)$ & $p$ value & $\beta$ & $95 \% \mathrm{CI}$ & $\exp (\beta)$ & $p$ value \\
\hline Gender & 0.221 & $(-0.077,0.518)$ & 1.247 & 0.146 & 0.405 & $(0.240,0.570)$ & 1.499 & 0.109 \\
\hline Age & 0.014 & $(0.009,0.020)$ & 1.005 & 0.083 & -0.039 & $(-0.061,-0.017)$ & 0.962 & 0.001 \\
\hline Follow-up time & 0.015 & $(0.002,0.027)$ & 1.015 & 0.012 & 0.004 & $(-0.033,0.041)$ & 1.004 & 0.031 \\
\hline $\mathrm{K} 2$ & -0.131 & $(-0.656,0.394)$ & 0.877 & 0.625 & -0.149 & $(-0.264,-0.034)$ & 0.862 & 0.211 \\
\hline $\mathrm{ACD}$ & -0.035 & $(-0.431,0.360)$ & 0.965 & 0.861 & 0.301 & $(-0.130,0.731)$ & 1.351 & 0.172 \\
\hline PD & -0.090 & $(-0.176,-0.004)$ & 0.914 & 0.141 & -0.102 & $(-0.163,-0.042)$ & 0.903 & 0.001 \\
\hline $\mathrm{CD}$ & 0.191 & $(-0.079,0.461)$ & 1.211 & 0.165 & -0.787 & $(-1.121,-0.453)$ & 0.455 & $<0.001$ \\
\hline IOP & -0.013 & $(-0.040,0.013)$ & 0.987 & 0.328 & 0.015 & $(-0.004,0.034)$ & 1.015 & 0.130 \\
\hline Wearing OK lens & -0.286 & $(-0.202,-0.370)$ & 1.247 & $<0.001$ & & & & \\
\hline
\end{tabular}

SER, spherical equivalent refractive; K1, the refraction measured on the flattest curve of the cornea; K2, the refraction measured on the deepest curve of the cornea; ACD, anterior chamber depth; PD, pupil diameter; $\mathrm{CD}$, corneal diameter; IOP, intraocular pressure; $\mathrm{AL}$, axial length; OK, orthokeratology; CI, confidence interval.

At the end of the treatment, the AL difference has decreased by $0.50 \pm 0.29 \mathrm{~mm}$, from $1.08 \pm 0.35 \mathrm{~mm}$ at baseline to $0.58 \pm 0.25 \mathrm{~mm}$ at the 24 -month visit $(F=24.539$, $p<0.001$; Table 2; Fig. 2, 3).

\section{Part Two}

Patient Characteristics with Binocular OK Lenses

We reviewed the data of 131 myopic anisometropia patients who used binocular OK lenses. The average initial wearing age was $12.92 \pm 2.60$ years (from 8 to 16 years), and the mean follow-up duration was $17.83 \pm 5.02$ months (from 7 to 26 months). Moreover, the average initial AL was significantly longer in the G group (25.42 $\pm 1.06 \mathrm{~mm})$ than that in the L group $(24.87 \pm 1.09 \mathrm{~mm})$ $(p<0.001)$. The average SER was $-4.79 \pm 1.90 \mathrm{D}$ in the $\mathrm{G}$ group and $-3.14 \pm 1.88 \mathrm{D}$ in the $\mathrm{L}$ group $(p<0.001)$. The baseline biometric data for the $\mathrm{G}$ group and L group obtained immediately before the children began wearing the lenses are presented in Table 4.

\section{AL and AL Elongation of the G Group and L Group}

The baseline AL values of the $\mathrm{G}$ and $\mathrm{L}$ groups were $25.42 \pm 1.06$ and $24.87 \pm 1.09 \mathrm{~mm}$, respectively $(p<0.001)$. After 24 months of follow-up, the G group's mean AL was $25.59 \pm 1.10 \mathrm{~mm}$ compared with $25.11 \pm 1.15 \mathrm{~mm}$ in the L group $(p<0.001)$.

Overall, the AL elongation in the G group was slower than that in the L group at the end of the 24-month study 
Fig. 2. The changes of AL difference in part one during follow-up time. Forty-seven monocular myopic subjects were involved in the study. Subjects with decreased AL difference were represented as black lines and those with increased AL difference were represented as red lines. There were 40 subjects who had decreased AL difference, and 7 subjects had increased AL difference. AL, axial length.

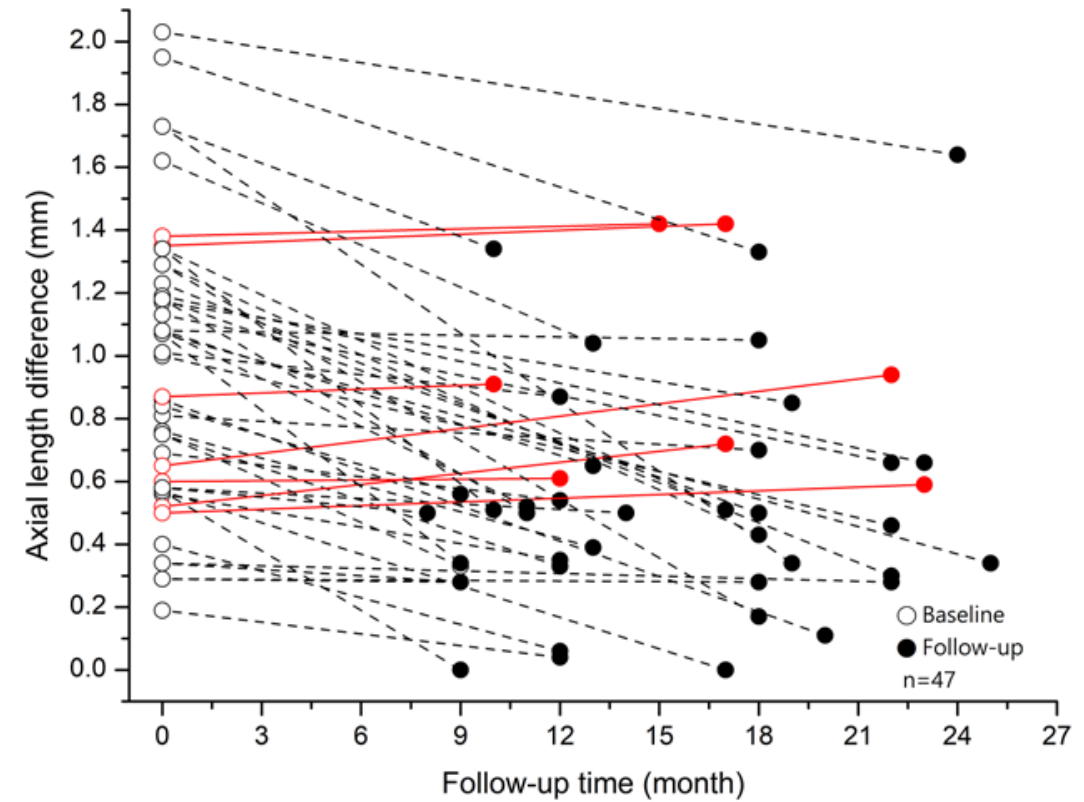

Follow-up time (month)
Table 4. Summary of the demographic data of the L group and the G group

\begin{tabular}{lccr}
\hline Variable & L group & G group & $p$ value \\
\hline Gender, $n(\%)$ & & & \\
$\quad$ Male & $56(42.7)$ & & \\
$\quad$ Female & $75(57.3)$ & & \\
Wearing age, years & $12.92 \pm 2.60(8-16)$ & & \\
Follow-up time, months & $17.83 \pm 5.02(7-26)$ & & \\
Side & & & \\
$\quad$ Right & 45 & 86 & \\
$\quad$ Left & 86 & 45 & \\
SERE, D & $-3.14 \pm 1.88$ & $-4.79 \pm 1.90$ & $<0.001^{*}$ \\
K1, D & $42.69 \pm 1.35$ & $42.78 \pm 1.30$ & $0.616^{*}$ \\
K2, D & $44.23 \pm 1.54$ & $43.89 \pm 3.79$ & $0.329^{*}$ \\
ACD, mm & $3.70 \pm 0.35$ & $3.74 \pm 0.35$ & $0.397^{*}$ \\
PD, mm & $6.16 \pm 0.78$ & $6.18 \pm 0.82$ & $0.906^{*}$ \\
CD, mm & $12.08 \pm 0.36$ & $12.08 \pm 0.33$ & $0.851^{*}$ \\
IOP, mm Hg & $16.97 \pm 2.47$ & $16.74 \pm 2.34$ & $0.491^{*}$ \\
AL, mm & $24.87 \pm 1.09$ & $25.42 \pm 1.06$ & $<0.001^{*}$ \\
& & & \\
& & &
\end{tabular}

SER, spherical equivalent refractive; K1, the refraction measured on the flattest curve of the cornea; K2, the refraction measured on the deepest curve of the cornea; ACD, anterior chamber depth; $\mathrm{PD}$, pupil diameter; $\mathrm{CD}$, corneal diameter; IOP, intraocular pressure; AL, axial length; $\mathrm{OK}$, orthokeratology; $\mathrm{D}$, diopter. $p$ value $<0.05$ was considered statistically significant after the test. ${ }^{*} p \leq$ 0.05 (obtained from independent $t$ tests).

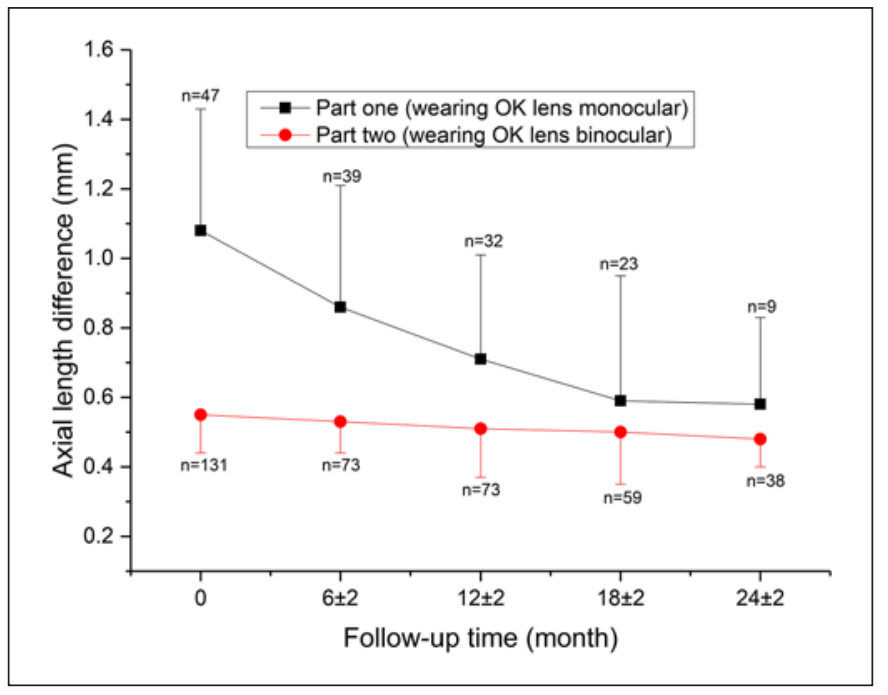

Fig. 3. The AL difference changes during follow-up time. Error bars represent standard errors. AL, axial length.

period. At 12 months, the L group's AL increased by 0.17 $\pm 0.11 \mathrm{~mm}$ compared with $0.13 \pm 0.11 \mathrm{~mm}(p=0.005)$ in the $\mathrm{G}$ group. In the $\mathrm{L}$ group, the $\mathrm{AL}$ elongation was 0.24 $\pm 0.18 \mathrm{~mm}$, while in the G group a lower AL elongation of $0.17 \pm 0.13 \mathrm{~mm}(p<0.001)$ was observed after 24 months. Thus, the L group's AL elongation was significantly greater than that of the G group at 24 months. The 
Table 5. The AL, AL elongation, and AL difference in the G group and L group

\begin{tabular}{|c|c|c|c|c|c|c|c|c|}
\hline & \multicolumn{3}{|l|}{$\mathrm{AL}, \mathrm{mm}$} & \multicolumn{3}{|c|}{ AL elongation, mm } & \multicolumn{2}{|c|}{ AL difference, $\mathrm{mm}$} \\
\hline & G group & L group & $p$ value & G group & L group & $p$ value & $\mathrm{AL}_{(\mathrm{G})}-\mathrm{AL}_{(\mathrm{L})}$ & $p$ value \\
\hline 6 months $(n=73)$ & $25.47 \pm 0.96$ & $24.94 \pm 1.00$ & $<0.001^{*}$ & $0.05 \pm 0.09$ & $0.07 \pm 0.13$ & $0.036^{*}$ & $0.53 \pm 0.09$ & $p=0.030^{\ddagger}$ \\
\hline 12 months $(n=73)$ & $25.55 \pm 0.92$ & $25.04 \pm 0.93$ & $<0.001^{*}$ & $0.13 \pm 0.11$ & $0.17 \pm 0.11$ & $0.005^{*}$ & $0.51 \pm 0.14$ & \\
\hline 18 months $(n=59)$ & $25.57 \pm 0.92$ & $25.07 \pm 0.92$ & $<0.001^{*}$ & $0.15 \pm 0.20$ & $0.20 \pm 0.12$ & $0.012^{*}$ & $0.50 \pm 0.15$ & \\
\hline
\end{tabular}

ANOVA, analysis of variance; AL, axial length. $p$ value $<0.05$ was considered statistically significant after the test. ${ }^{*} p$ obtained from an independent $t$ test. ${ }^{\ddagger} p$ obtained from repeated-measures ANOVA.

Table 6. The associations between the change of AL difference and other parameters

\begin{tabular}{lrllr}
\hline Predictors & Part two & & \\
\cline { 2 - 5 } & $\beta$ & $95 \%$ CI & $\exp (\beta)$ & $p$ value \\
\hline Intercept & 1.061 & $(0.384,1.737)$ & 2.888 & 0.002 \\
Gender & 0.218 & $(0.123,0.313)$ & 1.244 & 0.162 \\
Follow-up time & -0.027 & $(-0.053,-0.001)$ & 0.973 & 0.046 \\
Age & -0.009 & $(-0.031,0.014)$ & 0.991 & 0.456 \\
AL elongation in G group & -0.346 & $(-0.705,0.014)$ & 0.708 & 0.030 \\
AL elongation in L group & -0.081 & $(-0.381,0.218)$ & 0.922 & 0.595 \\
Initial SER difference & 0.404 & $(0.149,0.659)$ & 1.498 & 0.002 \\
Initial AL difference & 0.342 & $(0.249,0.435)$ & 1.408 & $<0.001$ \\
Initial K1 difference & -0.139 & $(-0.302,0.025)$ & 0.870 & 0.096 \\
Initial K2 difference & 0.118 & $(-0.039,0.276)$ & 1.126 & 0.141 \\
Initial ACD difference & 0.417 & $(0.099,0.735)$ & 1.517 & 0.210 \\
Initial PD difference & 0.050 & $(-0.052,0.151)$ & 1.051 & 0.336 \\
Initial CD difference & -0.020 & $(-0.026,-0.014)$ & 0.980 & 0.127 \\
Initial IOP difference & 0.014 & $(-0.007,0.036)$ & 1.014 & 0.196 \\
Scale & 0.018 & & & \\
\hline
\end{tabular}

SER, spherical equivalent refractive; $\mathrm{K} 1$, the refraction measured on the flattest curve of the cornea; K2, the refraction measured on the deepest curve of the cornea; ACD, central anterior chamber depth; PD, pupil diameter; CD, corneal diameter; IOP, intraocular pressure; AL, axial length; OK, orthokeratology.
$\mathrm{AL}$ and $\mathrm{AL}$ elongation data at each visit are presented in Table 5 and Figure 4.

In the GEE model, the variable factors of gender, keratometry, ACD, and IOP exhibited no effect on AL elongation $(p>0.05)$. Age, follow-up time, SER, PD, and CD were closely related to AL elongation ( $p<0.05$, Table 3$)$. Follow-up time, AL elongation in the G group, initial SER difference, and AL difference were associated with the change of AL difference $(p<0.05$, Table 6$)$.

AL Difference Decreased during the Study Period

After 12 months of treatment, a slight decrease in the AL difference of $0.04 \pm 0.13 \mathrm{~mm}$ was observed, from 0.55 $\pm 0.11 \mathrm{~mm}$ at baseline to $0.51 \pm 0.14 \mathrm{~mm}$ at the 12 months visit. At the end of the treatment, the AL difference had decreased by $0.07 \pm 0.09 \mathrm{~mm}$, from $0.55 \pm 0.11 \mathrm{~mm}$ at baseline to $0.48 \pm 0.08 \mathrm{~mm}$ at the 24 months visit $(F=$ $3.884, p=0.030$ ). During the study period, the AL difference decreased significantly (Table 5; Fig. 3).

\section{Discussion}

In myopic anisometropic patients, the AL of both myopic eyes continues to grow despite proper optical correction [3]. The OK lens is not only capable of providing an 


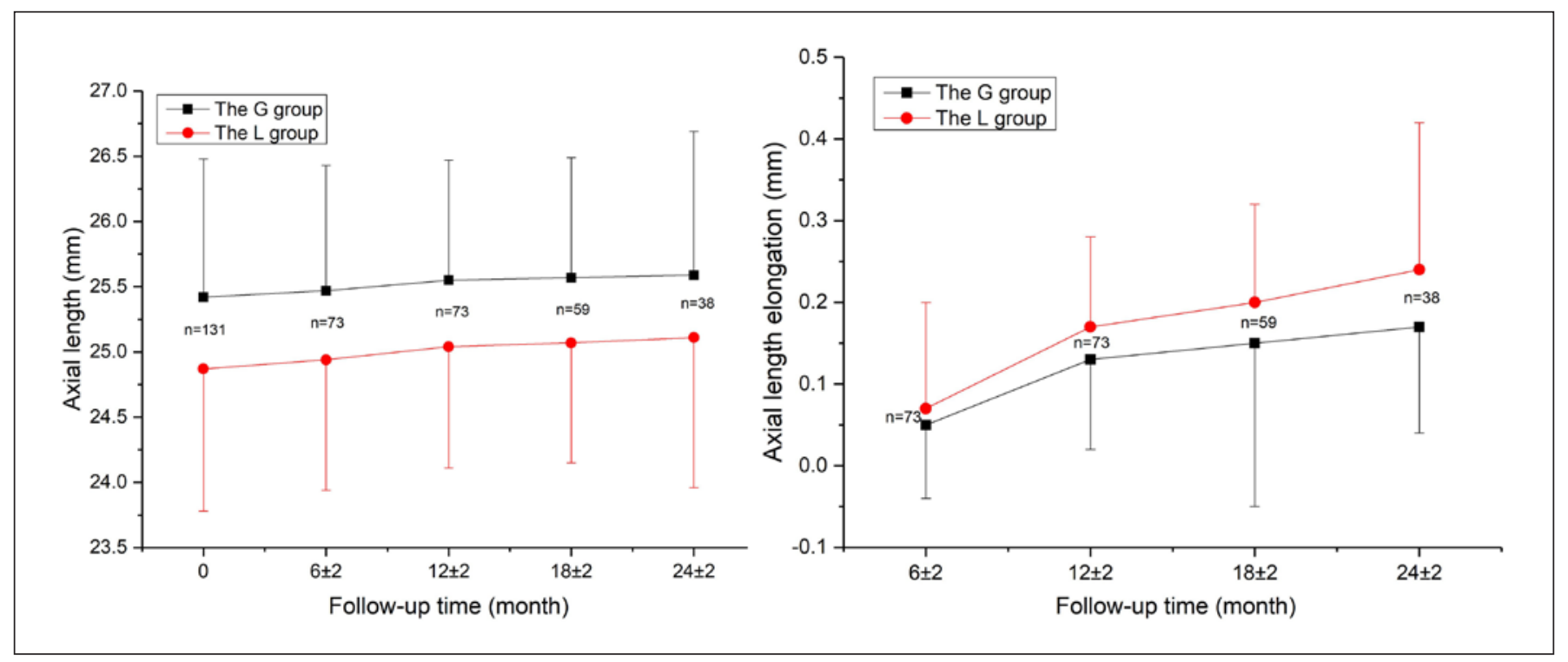

Fig. 4. Changes in AL and AL elongation of the G group and the L group. Error bars represent standard errors. AL, axial length.

optical correction but also of slowing down AL growth of treated eyes, resulting in a retardation of the magnitude of anisometropia, hence making it the treatment of choice for myopic anisometropic patients.

Several longitudinal studies have examined the development of anisometropia during childhood and typically reported an increase in the magnitude of interocular difference in refraction with age $[1,41-43]$. Since the chance of spontaneously occurring anisometropia decreasing with age is slim, the slowing of eye growth after OK lens treatment may be largely explained as an effect of the treatment modality alone.

In part one, we reviewed the data of 47 monocular myopic patients who used the monocular OK lenses. Although our conclusions were consistent with other studies, the changes of AL difference were quite distinct. For example, Fu et al. [44] reported an increase in AL of 0.11 $\pm 0.19 \mathrm{~mm}$ in the OK group and $0.30 \pm 0.28 \mathrm{~mm}$ in the control group over a one-year period; the AL difference decreased by $0.19 \mathrm{~mm}$. Long et al. [45] reported that the increase in $\mathrm{AL}$ after 1 year was $0.05 \pm 0.19 \mathrm{~mm}$ in the $\mathrm{OK}$ group and $0.34 \pm 0.21 \mathrm{~mm}$ in the control group; the $\mathrm{AL}$ difference had a decrease of $0.29 \mathrm{~mm}$. Na et al. [38] also reported a one-year AL increase of $0.07 \pm 0.21 \mathrm{~mm}$ in the OK group and $0.36 \pm 0.23 \mathrm{~mm}$ in the control group; the AL difference decreased to $0.29 \mathrm{~mm}$. Whereas, Chen et al. [46] reported a difference of $0.42 \mathrm{~mm}$ in AL elongation between myopic and nonmyopic eyes after wearing OK lenses for 12 months. In our study, the differences of $\mathrm{AL}$ elongation were $0.36 \pm 0.25 \mathrm{~mm}$ at 12 months and $0.50 \pm$ $0.29 \mathrm{~mm}$ at 24 months. There are several possible reasons for this discrepancy. This study has suggested that there is an uncontrolled variable called "intersubject difference" as well as the differences in the baseline between individuals who did and did not wear the OK lens. At the same time, it is likely that during the follow-up periods, the subjects will almost never live in the same environment [47]. In addition, some environmental factors could accelerate myopic progression of some children, such as excessive near work. The high value placed on educational performance is driving children to spend longer time in school and on their studies. Furthermore, there may be a selection bias for this study population. According to our clinical experience, children who had a rapid progression of myopia are more willing to choose OK lenses for controlling myopia.

However, the AL difference of 7 subjects increased during the wearing period, indicating that the AL elongation in the OK group increased more than that of the control group. This phenomenon has also appeared in the study of Chen et al. [46]. We have searched the PubMed studies and summarized several reasons. First, among the 7 monocular myopic subjects with the AL difference increased during follow-up time, there were 6 subjects who had mixed anisometropia (the eye in control group was hyperopia). There may be asymmetrical axial growth in both eyes and leads to an increase in the AL difference. Second, partial subjects were less compli- 
ant in wearing OK lenses; the insufficient time spent by subjects in wearing OK lenses, possibly due to a lack of discipline and care, may reduce the effectiveness of OK lenses [48-50]. Studies had shown that with OK lens treatment, boys had greater AL elongation than girls. The reason may be attributed to anecdotal accounts indicating that boys are less compliant than girls in wearing $\mathrm{OK}$ lenses [51, 52]. A study by Morgan et al. [53] showed that compliance decreases with age. Radford et al. [54] also found that compliance decreased rapidly within the first 2 years, with a slower rate of deterioration in hygiene compliance thereafter. Third, the parental involvement may be another reason. For some children who were unable to wear OK lenses by themselves, they would be reluctant to wear the lenses because of frustrating feelings during the wearing procedure. Furthermore, due to their unfamiliarity with the procedures, the lenses could drop or break, which demanded additional time. Sometimes the parents may stop encouraging them to wear the lenses due to the children's routines and lack of motivation. This was especially true when parents believed that their children did not need to rely on their eyesight, such as during winter and summer vacation, when children did not wear the lenses daily [50]. Fourth, the follow-up time may be the reason for fluctuation of the AL difference during the study period. Statistically significantly higher AL growth during the winter period was observed [55, 56]. In the study of Donovan et al. [57], myopia progression in summer months was approximately $60 \%$ of that seen in winter. Based on cycloplegic autorefraction over a 10 -month period, it was found that, in 7-year-olds, there was a significant increase in the rate of myopia progression after the academically important final examinations. This period of increased progression coincided with the summer vacation at the end of the academic year [55]. When they had greater academic pressure or faced the important entrance examination, they may give up wearing OK lenses. Finally, our study has limitations considering its retrospective nature, small sample size, factors that may affect myopic progression, and living habits were not considered in our study.

In part 2, we reviewed the data of 131 myopic anisometropia patients who used the OK lenses as binoculars. This study has demonstrated that OK lenses were more effective for myopia management in eyes with more severe myopia than in eyes with more moderate degrees of myopia. Previous studies have reported that AL growth was positively correlated with baseline SER $[25,58]$. Other clinical trials also demonstrated that OK lens wear has a more significant effect on slowing AL elongation in the

Effects of Orthokeratology on

Anisometropes more myopic eye of anisometropic patients. Zhong et al. [58] reviewed 29 cases of myopic anisometropia and discovered that after 24 months of follow-up, the AL had increased by $0.31 \pm 0.23 \mathrm{~mm}$ in the more myopic eyes with higher baseline values and by $0.41 \pm 0.31 \mathrm{~mm}$ in the contralateral eyes. Notwithstanding, Fu et al. [44] found that OK lenses can control the AL elongation in both eyes at the same rate, and it cannot reduce the degree of anisometropia in children with bilateral myopia and concurrent anisometropia. As a result, we may need prospective experiments and more extensive experimental data to further verify the relationship between the therapeutic effects of OK lenses and SER.

According to the results of the GEE model in part 2, age has a significant impact on AL elongation. This may be attributed to the fact that the AL is related to the initial age at baseline, and studies have revealed that AL elongation slows with age $[59,60]$. Hyman et al. [61] found that the rate of myopic progression decreased with age and stopped after the age of 15 years in males and 14 years in females. The greatest change in myopia in Chinese children was reported in those ranging in age 9-11 years [62]. Hiraoka et al. [63] confirmed that younger children benefited more from Ortho-K treatment than older children. $\mathrm{PD}$ was negatively correlated with AL elongation. Chen et al. [64] demonstrated that large PDs promote the effect of Ortho-K to slow axial growth in myopia; they speculated that this is because of enhancement of the myopic shift in the peripheral retina. In part 2, the change of $\mathrm{AL}$ difference was negatively correlated with the increase of $\mathrm{AL}$ elongation in the G group.

In this study, we used the same patient's contralateral eye as a control group, comprising the same genetic inheritance. This study design minimized the sample size for paired analysis without sacrificing the statistical power and limited the risks of attrition bias from study dropouts. Nevertheless, our study has limitations considering its retrospective nature. Even though we used contralateral nonmyopic eyes as a control group, it would be more accurate to use contact lenses without any therapeutic effect on those eyes. In addition, the OK lenses themselves somehow affect corneal epithelium shortening. Thus, it would be more accurate to measure a baseline AL before applying OK lenses and then establishing a comparison with the subsequent data. Besides, factors that may affect myopic progression, such as peripheral refractive status, accommodative lag, and retinal image quality, were not recorded in either group. So, additional prospective studies with more participants are necessary. 
In conclusion, monocular OK lenses were effective in suppressing the AL growth of myopic eyes and reducing the severity of anisometropia in unilateral myopic children. The OK lenses are also capable of slowing down $\mathrm{AL}$ growth in bilateral myopic anisometropes and have a greater effect on lessening AL elongation in the more myopic eye of anisometropic patients.

\section{Statement of Ethics}

The study was conducted in accordance with the principles of the Declaration of Helsinki, and the study protocol was approved by the Ethics Committee of the Affiliated Eye Hospital of Shandong University of Traditional Chinese Medicine with approval No (HEC-KS-2020001KY). Because of the retrospective nature of the study, patient consent for inclusion was waived.

\section{Conflict of Interest Statement}

The authors declare no conflicts of interest. The authors alone are responsible for the content and writing of this article.

\section{Funding Sources}

This work was supported by the Chinese National Key Research and Development Project (2019YFC1710200, 2019YFC1710203).

\section{Author Contributions}

Jing $\mathrm{Xu}$ contributed to acquisition of data, analysis and interpretation of data, and drafting the manuscript; Bei Gao contributed to acquisition of data, analysis, and interpretation of data; Qingmei Tian contributed to analysis and interpretation of data and obtaining funding; Qiuxin Wu contributed to critical revision for intellectual content; Xiuyan Zhang contributed to analysis and interpretation of data; Xiao Lin contributed to conception and design; Ruixue Zhang contributed to analysis and interpretation of data; Jike Song contributed to conception and design, drafting the manuscript, critical revision for intellectual content, and obtaining funding; Hongsheng Bi contributed to conception and design and obtaining funding.

\section{Data Availability Statement}

The datasets used and analyzed during the current study are available from the corresponding author on reasonable request.

\section{References}

1 Deng L, Gwiazda JE. Anisometropia in children from infancy to 15 years. Invest Ophthalmol Vis Sci. 2012;53:3782-7.

2 Jiang Z, Shen M, Xie R, Qu J, Xue A, Lu F. Interocular evaluation of axial length and retinal thickness in people with myopic anisometropia. Eye Contact Lens. 2013;39:277-82.

3 Weakley DR Jr. The association between nonstrabismic anisometropia, amblyopia, and subnormal binocularity. Ophthalmology. 2001;108:163-71.

4 Li SM, Ji YZ, Wu SS, Zhan SY, Wang B, Liu $\mathrm{LR}$, et al. Multifocal versus single vision lenses intervention to slow progression of myopia in school-age children: a meta-analysis. Surv Ophthalmol. 2011;56:451-60.

5 Karimian F, Ownagh V, Amiri MA, Tabatabaee SM, Dadbin N. Stereoacuity after wavefront-guided photorefractive keratectomy in anisometropia. J Ophthalmic Vis Res. 2017; 12:265-9.

6 Lovasik JV, Szymkiw M. Effects of aniseikonia, anisometropia, accommodation, retinal illuminance, and pupil size on stereopsis. Invest Ophthalmol Vis Sci. 1985;26:741-50.

7 Borchert M, Tarczy-Hornoch K, Cotter SA, Liu N, Azen SP, Varma R, et al. Anisometropia in Hispanic and African American infants and young children the multi-ethnic pediatric eye disease study. Ophthalmology. 2010;117: 148-53.e1.

8 Huynh SC, Wang XY, Ip J, Robaei D, Kifley A, Rose KA, et al. Prevalence and associations of anisometropia and aniso-astigmatism in a population based sample of 6 year old children. Br J Ophthalmol. 2006;90:597-601.

9 Almeder LM, Peck LB, Howland HC. Prevalence of anisometropia in volunteer laboratory and school screening populations. Invest Ophthalmol Vis Sci. 1990;31:2448-55.

10 Yekta A, Fotouhi A, Hashemi H, Dehghani C, Ostadimoghaddam H, Heravian J, et al. The prevalence of anisometropia, amblyopia and strabismus in schoolchildren of Shiraz. Iran. Strabismus. 2010;18:104-10.

11 Yamashita T, Watanabe S, Ohba N. A longitudinal study of cycloplegic refraction in a cohort of 350 Japanese schoolchildren. Anisometropia. Ophthalmic Physiol Opt. 1999;19: $30-3$.

12 Liang YB, Wong TY, Sun LP, Tao QS, Wang JJ, Yang XH, et al. Refractive errors in a rural Chinese adult population the Handan eye study. Ophthalmology. 2009;116:2119-27.

13 Guzowski M, Fraser-Bell S, Rochtchina E, Wang JJ, Mitchell P. Asymmetric refraction in an older population: the Blue Mountains Eye Study. Am J Ophthalmol. 2003;136:5513.

14 Qin XJ, Margrain TH, To CH, Bromham N, Guggenheim JA. Anisometropia is independently associated with both spherical and cylindrical ametropia. Invest Ophthalmol Vis Sci. 2005;46:4024-31.

15 Zhong X, Ge J, Nie H, Smith EL 3rd. Compensation for experimentally induced hyperopic anisometropia in adolescent monkeys. Invest Ophthalmol Vis Sci. 2004;45:3373-9.

16 Siegwart JT Jr, Norton TT. Binocular lens treatment in tree shrews: effect of age and comparison of plus lens wear with recovery from minus lens-induced myopia. Exp Eye Res. 2010;91:660-9.

17 Zaka-Ur-Rab S. Evaluation of relationship of ocular parameters and depth of anisometropic amblyopia with the degree of anisometropia. Indian J Ophthalmol. 2006;54:99-103.

18 Tian Y, Tarrant J, Wildsoet CF. Optical and biometric characteristics of anisomyopia in human adults. Ophthalmic Physiol Opt. 2011; 31:540-9.

19 Kee CS, Deng L. Astigmatism associated with experimentally induced myopia or hyperopia in chickens. Invest Ophthalmol Vis Sci. 2008; 49:858-67.

20 Larsson EK, Holmström GE. Development of astigmatism and anisometropia in preterm children during the first 10 years of life: a population-based study. Arch Ophthalmol. 2006; 124:1608-14.

21 Saunders KJ, McCulloch DL, Shepherd AJ, Wilkinson AG. Emmetropisation following preterm birth. Br J Ophthalmol. 2002;86: 1035-40.

22 Hashemi H, Khabazkhoob M, Yekta A, Mohammad K, Fotouhi A. Prevalence and risk factors for anisometropia in the Tehran Eye Study, Iran. Ophthalmic Epidemiol. 2011;18: 122-8. 
23 Li SM, Kang MT, Wu SS, Meng B, Sun YY, Wei SF, et al. Studies using concentric ring bifocal and peripheral add multifocal contact lenses to slow myopia progression in schoolaged children: a meta-analysis. Ophthalmic Physiol Opt. 2017;37:51-9.

24 Song YY, Wang H, Wang BS, Qi H, Rong ZX, Chen HZ. Atropine in ameliorating the progression of myopia in children with mild to moderate myopia: a meta-analysis of controlled clinical trials. J Ocul Pharmacol Ther. 2011;27:361-8.

25 Kakita T, Hiraoka T, Oshika T. Influence of overnight orthokeratology on axial elongation in childhood myopia. Invest Ophthalmol Vis Sci. 2011;52(5):2170-4.

26 Lithander J, Sjöstrand J. Anisometropia and strabismic amblyopia in the age group two years and above: a prospective study of the results of treatment. Br J Ophthalmol. 1991;75: 111-6.

27 O'Keefe M, Nolan L. LASIK surgery in children. Br J Ophthalmol. 2004;88:19-21.

28 Yin ZQ, Wang H, Yu T, Ren Q, Chen L. Facilitation of amblyopia management by laser in situ keratomileusis in high anisometropic hyperopic and myopic children. J AAPOS. 2007;11:571-6

29 Alió JL, Wolter NV, Piñero DP, Amparo F, Sari ES, Cankaya C, et al. Pediatric refractive surgery and its role in the treatment of amblyopia: meta-analysis of the peer-reviewed literature. J Refract Surg. 2011;27:364-74.

30 Salmon T, van de Pol C. Normal-eye Zernike. Coefficients and root-mean-square wavefront errors. J Cataract Refractive Surg. 2006; 32:2064-74.

31 Cheng H, Barnett JK, Vilupuru AS, Marsack JD, Kasthurirangan S, Applegate RA, et al. A population study on changes in wave aberrations with accommodation. J Vis. 2004;4: 272-80.

32 Charman WN, Mountford J, Atchison DA, Markwell EL. Peripheral refraction in orthokeratology patients. Optom Vis Sci. 2006;83: 641-8.

33 Kang P, Swarbrick H. Peripheral refraction in myopic children wearing orthokeratology and gas-permeable lenses. Optom Vis Sci. 2011;88:476-82.

34 Smith EL III. Prentice Award Lecture 2010: a case for peripheral optical treatment strategies for myopia. Optom Vis Sci. 2011;88: 1029-44.

35 Cho P, Cheung SW. Retardation of Myopia in Orthokeratology (ROMIO) Study: a 2-year randomized clinical trial. Invest Ophthalmol Vis Sci. 2012;53:7077-85.
36 Swarbrick HA, Alharbi A, Watt K, Lum E, Kang P. Myopia control during orthokeratology lens wear in children using a novel study design. Ophthalmology. 2015;122:620-30.

37 Charm J, Cho P. High myopia-partial reduction Ortho-K: a 2-year randomized study. Optom Vis Sci. 2013;90:530-9.

$38 \mathrm{Na} \mathrm{M}$, Yoo A. The effect of orthokeratology on axial length elongation in children with myopia: contralateral comparison study. Jpn J Ophthalmol. 2018;62:327-34.

39 Lum E. Progressive anisometropia and orthokeratology: a case report. Clin Exp Optom. 2018;101:599-600.

40 Tsai WS, Wang JH, Lee YC, Chiu CJ. Assessing the change of anisometropia in unilateral myopic children receiving monocular orthokeratology treatment. J Formos Med Assoc. 2019;118:1122-8.

41 Parssinen O. Anisometropia and changes in anisometropia in school myopia. Optom Vis Sci. 1990;67:256-9.

42 Tong L, Chan YH, Gazzard G, Tan D, Saw SM. Longitudinal study of anisometropia in Singaporean school children. Invest Ophthalmol Vis Sci. 2006;47:3247-52.

43 Pointer JS, Gilmartin B. Clinical characteristics of unilateral myopic anisometropia in a juvenile optometric practice population. Ophthalmic Physiol Opt. 2004;24:458-63.

$44 \mathrm{Fu} \mathrm{AC}$, Qin J, Rong JB, Ji N, Wang WQ, Zhao $\mathrm{BX}$, et al. Effects of orthokeratology lens on axial length elongation in unilateral myopia and bilateral myopia with anisometropia children. Cont Lens Anterior Eye. 2020;43:73-7.

45 Long W, Li Z, Hu Y, Cui D, Zhai Z, Yang X. Pattern of axial length growth in children myopicanisometropes with orthokeratology treatment. Curr Eye Res. 2020;45:834-8.

46 Chen Z, Zhou J, Qu X, Zhou X, Xue F. Shanghai Orthokeratology Study (SOS) Group. Effects of orthokeratology on axial length growth in myopic anisometropes. Contact Lens Anterior Eye. 2018;41:263-6.

47 Chan KY, Cheung SW, Cho P. Orthokeratology for slowing myopic progression in a pair of identical twins. Cont Lens Anterior Eye. 2014;37:116-9.

48 Santolaria E, Cerviño A, Queirós A, Brautaset $\mathrm{R}$, González-Méijome JM. Subjective satisfaction in long-term orthokeratology patients. Eye Contact Lens. 2013;39(6):388-93.

49 Jun J, Zhiwen B, Feifu W, Lili L, Fan L. Level of compliance in orthokeratology. Eye Contact Lens. 2018;44(5):330-4.

50 Chang LC, Li FJ, Sun CC, Liao LL. Trajectories of myopia control and orthokeratology compliance among parents with myopic children. Cont Lens Anterior Eye. 2021 Aug; 44(4):101360
51 Yu LH, Jin WQ, Mao XJ, Jiang J. Effect of orthokeratology on axial length elongation in moderate myopic and fellow high myopic eyes of children. Clin Exp Optom. 2021 Jan; 104(1):22-7.

52 Santodomingo-Rubido J, Villa-Collar C, Gilmartin B, Gutiérrez-Ortega R. Factors preventing myopia progression with orthokeratology correction. Optom Vis Sci. 2013; 90(11):1225-36.

53 Morgan PB, Efron N, Toshida H, Nichols JJ. An international analysis of contact lens compliance. Cont Lens Anterior Eye. 2011;34(5): 223-8.

54 Radford CF, Woodward EG, Stapleton F. Contact lens hygiene compliance in a universitypopulation. J Br Contact Lens Assoc. 1993; 16(3):105-11.

55 Rusnak S, Salcman V, Hecova L, Kasl Z. Myopia progression risk: seasonal and lifestyle variations in axial length growth in Czech children. J Ophthalmol. 2018;2018:5076454.

56 Fulk GW, Cyert LA, Parker DA. Seasonal variation in myopia progression and ocular elongation. Optom Vis Sci. 2002;79(1):46-51.

57 Donovan L, Sankaridurg P, Ho A, Chen X, Lin $\mathrm{Z}$, Thomas $\mathrm{V}$, et al. Myopia progression in Chinese children is slower in summer than in winter. Optom Vis Sci. 2012;89(8):1196-202.

58 Zhong Y, Ke L, Qiong W, Liu F. Orthokeratology lens for management of myopia in anisometropic children: a contralateral study. Cont Lens Anterior Eye. 2020;43:40-3.

59 Fan DS, Lam DS, Lam RF, Lau JT, Chong KS Cheung EY, et al. Prevalence, incidence, and progression of myopia of school children in Hong Kong. Invest Ophthalmol Vis Sci. 2004; 45:1071-5.

60 Edwards MH. The development of myopia in Hong Kong children between the ages of 7 and 12 years: a five-year longitudinal study. Ophthalmic Physiol Opt. 1999;19:286-94.

61 Hyman L, Gwiazda J, Hussein M, Norton TT, Wang Y, Marsh-Tootle W, et al. Relationship of age, sex, and ethnicity with myopia progression and axial elongation in the correction of myopia evaluation trial. Arch Ophthalmol. 2005;123:977-87.

62 Goss DA, Winkler RL. Progression of myopia in youth: age of cessation. Am J Optom Physiol Opt. 1983;60:651-8.

63 Hiraoka T, Kakita T, Okamoto F, Takahashi $\mathrm{H}$, Oshika T. Long-term effect of overnight orthokeratology on axial length elongation in childhood myopia: a 5-year follow-up study. Invest Ophthalmol Vis Sci. 2012;53:3913-9.

64 Chen Z, Niu L, Xue F, Qu X, Zhou Z, Zhou X, et al. Impact of pupil diameter on axial growth in orthokeratology. Optom Vis Sci. 2012;89: $1636-40$
Effects of Orthokeratology on

Anisometropes
Ophthalmic Res 2021;64:991-1001 